\title{
Nonlinear Dynamics of the Human Smooth Pursuit System in Health and Disease: Model Structure and Parameter Estimation
}

\author{
Viktor Bro ${ }^{1}$ and Alexander V. Medvedev ${ }^{1}$
}

\begin{abstract}
Oculomotor tests (OMT) are administered to quantify symptoms in neurological and mental diseases. Eye movements in response to displayed visual stimuli are registered by an digital video-based eye tracker and processed. Stimuli of simple signal form, e.g. sine waves, are traditionally used in medical practice to test the performance of the oculomotor system in smooth pursuit (SP). The calculated SP gain and the phase shift at the frequency in question are then presented as the test outcome. This paper revisits the problem of quantifying the SP dynamics from eye-tracking data by means of nonlinear system identification. First, a sparse Volterra-Laguerre (VL) model is estimated from an OMT with sufficiently exciting (in frequency and amplitude) stimuli. Then the structure and initial parameter estimates of a polynomial Wiener model (WM) are obtained from the kernel estimates of the VL model. Finally, the parameter distributions of the WM are inferred by a particle filter (PF). In the proposed approach, the performance of the PF is improved by the individualized sparse model structure. Experimental data show that the latter captures the alternations in the SP dynamics due to aging and in Parkinson's disease.
\end{abstract}

\section{INTRODUCTION}

The particle filter (PF) algorithms have become a stateof-the-art technology in nonlinear estimation and, in particular, system identification [16]. Compared to the Extended Kalman Filter (see e.g. [10]), a PF does not approximate the system equations, but rather utilizes a particle set to capture the conditional distribution functions involved in the nonlinear state estimation problem. Further, the PF is not limited to stochastic variables with single-mode distribution since the particle set estimates the distribution and there is no need in reducing it to a point estimate, i.e. the expectation.

Despite the methodological benefits, the PF poses at least two implementation problems: First, the algorithm is computationally demanding and does not scale well when the number of estimated states grows. This shortcoming can be somehow alleviated by parallelization of the algorithm. Linear speed-up in the number of parallel cores can be achieved in several PF algorithms executed on a standard multicore platform, [15]. Second, the PF relies on a perfect knowledge of the process model and attempts to capture model uncertainty as an effect of noise. Notice also that the standard PF algorithms lack explicit feedback and substitute it by resampling procedures. In e.g. the EKF, model uncertainty can be attenuated by the output error feedback.

\footnotetext{
*This work was partly supported by Vinnova, Sweden's Innovation Agency through the project "Multimodal motor symptoms quantification platform for individualized Parkinson's disease treatment", MuSyQ.

${ }^{1}$ Division of Systems and Control, Department of Information Technology, Uppsala University, Uppsala, Sweden \{viktor.bro, alexander.medvedev\}@it.uu.se
}

A model structure is typically obtained in nonlinear identification from a priori information about the operational principles and organization of the system. Besides, detailed mathematical (simulation or first-principles) models of systems in technology are often readily available already at the system design stage, i.e. before the actual system is built.

However, in biomedical systems, the mechanisms behind many phenomena are not well-understood and the very purpose of biological control loops is not always clear. Even though the PF has been successfully used e.g. for the estimation of pharmacokinetic-pharmacodynamic models in closed-loop anesthesia [12], the model structure in this application is well-known in pharmacometrics.

In general, to enable the effective use of the PF algorithms in estimating mathematical models of biological and medical systems, a framework for model structure evaluation is necessary. This paper proposes to employ sparse Volterra-Laguerre (VL) modeling in order to capture nonlinear dynamics from an input-output data set. Then, a polynomial Wiener model (WM) can be estimated from the kernel Laguerre spectra of the Volterra model and serve as a model structure in a PF identification algorithm. This multi-step procedure combines the black-box properties of VL modeling with the general Bayesian nonlinear estimation framework of the PF without sacrificing computational feasibility.

All the individual steps of the proposed nonlinear identification approach are described in recent publications but have never been utilized together. The main practical contribution of this work is in the application of the proposed concept to modeling of the human smooth pursuit system (SPS).

The paper is composed as follows. First, the human SPS is described. A summary of the VL model is then provided, along with an overview of the Wiener model. State estimation using the PF is also outlined. Further, the experimental setup used this paper is described. Model estimation results from eye-tracking data are then presented, using a polynomial WM whose structure is obtained from a sparse VL model and parameters are estimated both using ordinary least squares and the PF. Finally, conclusions are drawn in Section VI.

\section{PRELIMINARIES}

\section{A. The human smooth pursuit system}

Smooth pursuit and saccades are the two main ways that humans shift their gaze in. Saccades are quick, episodic movements, while the SPS allows the gaze to continuously track some object of interest and keep it within focus. The SPS is a complex neurally controlled feedback system involving the eyes, the extraocular muscles, and several parts 
of the brain [19]. The SPS performance may thus be affected if any of these parts are compromised. Common examples include alcohol and drugs [20], as well as mental conditions, such as schizophrenia [13], and neurological conditions, e.g. Parkinson's disease [3], [4], [5], [6], [7], [8], [11].

\section{B. The Volterra model}

The Volterra series is a functional expansion of a dynamical, nonlinear, time-invariant system. A discrete system with input $u(k) \in \mathbb{R}$ and output $y(k) \in \mathbb{R}, k=0, \cdots, K-1$ may be approximated by the truncated Volterra series

$$
y(k)=y_{0}+\sum_{n=1}^{N} H_{n} u(k)+e(k),
$$

where $e(k) \in \mathbb{R}$ is the noise (or approximation error) term, $N \in \mathbb{N}$ is the Volterra order, and

$H_{n} u(k)=\sum_{i_{1}=0}^{\infty} \cdots \sum_{i_{n}=0}^{\infty} h_{n}\left(i_{1}, \cdots, i_{n}\right) u\left(k-i_{1}\right) \cdots u\left(k-i_{n}\right)$

are the Volterra functionals. The functions $h_{n}$ are called Volterra kernels and cumbersome to calculate explicitly. Therefore, the kernels are usually expanded in a functional basis. If the kernels $h_{n} \in \ell^{2}[0, \infty)$ and are not excessively oscillative, the discrete Laguerre functions can be used.

\section{Laguerre series representation of the Volterra Kernels}

The $j$ :th Laguerre function is defined in the $\mathcal{Z}$-domain as

$$
\Phi_{j}(z)=\frac{\sqrt{1-\alpha} z}{z-\sqrt{\alpha}}\left(\frac{1-\sqrt{\alpha} z}{z-\sqrt{\alpha}}\right)^{j},
$$

where $0<\alpha<1$ is the Laguerre parameter. The inverse $\mathcal{Z}$-transform yields the corresponding time-domain functions $\phi_{j}(k)=\mathcal{Z}^{-1}\left\{\Phi_{j}(z)\right\}$. These functions form an orthonormal basis in $\ell_{2}[0, \infty)$ so that any function $h(k) \in \ell_{2}[0, \infty)$ can be unambiguously written as a Laguerre series

$$
h(k)=\sum_{j=0}^{\infty} \gamma_{j} \phi_{j}(k) .
$$

The Volterra functionals can then be parametrized as

$$
\begin{aligned}
& h_{n}\left(i_{1}, \cdots, i_{n}\right)= \\
& \sum_{j_{1}=0}^{\infty} \cdots \sum_{j_{n}=0}^{\infty} \gamma_{n}\left(j_{1}, \cdots, j_{n}\right) \phi_{j_{1}}\left(i_{1}\right) \cdots \phi_{j_{n}}\left(i_{n}\right) .
\end{aligned}
$$

Only a finite number of $\phi_{j}(k)$ can be reliably calculated and a truncated Laguerre series of the true kernel is employed instead. A truncated series of the Laguerre order $L$ is then

$$
\begin{aligned}
& h_{n}\left(i_{1}, \cdots, i_{n}\right) \approx \\
& \sum_{j_{1}=0}^{L} \cdots \sum_{j_{n}=0}^{L} \gamma_{n}\left(j_{1}, \cdots, j_{n}\right) \phi_{j_{1}}\left(i_{1}\right) \cdots \phi_{j_{n}}\left(i_{n}\right),
\end{aligned}
$$

and the Volterra functionals become

$$
H_{n} u(k)=\sum_{j_{1}=0}^{\infty} \cdots \sum_{j_{n}=0}^{\infty} \gamma_{n}\left(j_{1}, \ldots, j_{n}\right) \psi_{j_{1}}(k) \cdots \psi_{j_{n}}(k),
$$

where $\psi_{j}(k)=\left(\phi_{j} * u\right)(k)=\sum_{l=0}^{k} \phi_{j}(l) u(k-l)$ denotes the Laguerre filter output and $(\cdot * \cdot)$ is the convolution operator. The VL model is finally written as

$y(k)=y_{0}+\sum_{n=1}^{N} \sum_{j_{1}=0}^{L} \cdots \sum_{j_{n}=0}^{L} \gamma_{n}\left(j_{1}, \ldots, j_{n}\right) \prod_{l=1}^{n} \psi_{j_{l}}+e(k)$.

Note however that the kernel functions are symmetric with respect to index, since the Laguerre functions commute. Many VL coefficients are therefore redundant and cannot be estimated individually. This reduces the number of coefficients in a Volterra model of order $N$ parametrized in $L$ Laguerre functions from $\left(L^{N+1}-1\right) /(L-1)$ to

$$
N_{c}=\left(\begin{array}{c}
L+N+1 \\
N
\end{array}\right)
$$

Now, a linear VL model (i.e. $N=1$ ) in state space is

$$
\begin{aligned}
\psi(k+1) & =\mathbf{F} \psi(k)+\mathbf{G} u(k), \\
y(k) & =\mathbf{c}^{T} \psi(k)+e(k),
\end{aligned}
$$

where $\mathbf{c}=\left[\begin{array}{lll}\gamma_{1}(0) & \gamma_{1}(1) & \ldots\end{array}\right]$ is a vector of VL parameters, $\psi(k)=\left[\begin{array}{lll}\psi_{0}(k) & \psi_{1}(k) & \ldots\end{array}\right]^{T}$ and

$\mathbf{F}=\left[\begin{array}{ccccc}\sqrt{\alpha} & 0 & 0 & & \\ 1-\alpha & \sqrt{\alpha} & 0 & \ldots & \ldots \\ -\sqrt{\alpha}(1-\alpha) & 1-\alpha & \sqrt{\alpha} & & \\ \sqrt{\alpha}^{2}(1-\alpha) & -\sqrt{\alpha}(1-\alpha) & 1-\alpha & \sqrt{\alpha} & \\ \vdots & \vdots & \vdots & \vdots & \ddots\end{array}\right]$

$$
\mathbf{G}=\sqrt{1-\alpha}\left[\begin{array}{lllll}
1 & -\sqrt{\alpha} & \sqrt{\alpha}^{2} & -\sqrt{\alpha}^{3} & \ldots
\end{array}\right]^{T} .
$$

\section{The Wiener model}

The Wiener model (WM) is a block-structured model compsrising a linear dynamic part cascaded with a static output nonlinearity. Consider a WM with the output polynomial nonlinearity $f_{m}(x)=x+d_{2} x^{2}+\cdots+d_{m} x^{m}$

$$
\begin{aligned}
\psi_{L}(k+1) & =\mathbf{F}_{L} \psi_{L}(k)+\mathbf{G}_{L} u(k), \\
y_{l}(k) & =\mathbf{c}^{T} \psi_{L}(k)+\varepsilon(k), \\
y(k) & =f_{m}\left(y_{l}(k)\right),
\end{aligned}
$$

where $\psi_{L}(k), \mathbf{F}_{L}$ and $\mathbf{G}_{L}$ are truncated versions of $\psi(k)$, $\mathbf{F}$ and $\mathbf{G}$, and $y_{l}$ is the output from the linear part of the model. The method of [9] for estimating the VL coefficients as well as the coefficients of (12) is recapitulated below.

The output in (12) can be expanded as

$$
\begin{aligned}
y(k) & =\mathbf{c}^{T} \psi_{L}(k)+d_{2}\left(\mathbf{c}^{T} \psi_{L}(k)\right)^{2}+\cdots \\
& +d_{m}\left(\mathbf{c}^{T} \psi_{L}(k)\right)^{m}+g(\varepsilon(k)) .
\end{aligned}
$$

This recasts in regressor form as $y=\varphi^{T} \gamma+g(\varepsilon)$, where

$$
\begin{aligned}
& \varphi=[\underbrace{1}_{\varphi_{0}} \underbrace{\psi_{L}^{T}}_{\varphi_{1}} \underbrace{\left(\psi_{L} \otimes \psi_{L}\right)^{T}}_{\varphi_{2}} \cdots \underbrace{}_{\varphi_{N}}]^{T} \\
& \gamma=[\underbrace{0}_{\gamma_{0}} \underbrace{\mathbf{c}^{T}}_{\gamma_{1}} \underbrace{d_{2}(\mathbf{c} \otimes \mathbf{c})^{T}}_{\gamma_{2}} \underbrace{d_{3}((\mathbf{c} \otimes \mathbf{c}) \otimes \mathbf{c})^{T}}_{\gamma_{3}} \ldots]^{T},
\end{aligned}
$$


$\otimes$ denotes the Kronecker product and $\psi_{L}=$ $\left[\psi_{L}(1) \psi_{L}(2) \ldots\right]$. Observing that $\varphi_{n}^{T} \gamma_{n}=d_{n}\left(\varphi_{1}^{T} \gamma_{1}\right)^{n}$, a scheme for estimating the linear coefficients $\mathbf{c}$ as well as the polynomial coefficients $\mathbf{d}=\left[\begin{array}{lll}d_{2} & d_{3} & \ldots\end{array}\right]^{T}$ is constructed from two sequential least squares estimation steps.

First, find an estimate $\hat{\gamma}$ of the VL coefficients by solving

$$
\hat{\gamma}=\arg \min _{\gamma} \sum_{k=1}^{K}\left|y(k)-\varphi^{T}(k) \gamma\right|^{2} \text {. }
$$

An estimate of the linear part is then $\hat{y}_{l}=\varphi_{1}^{T}(k) \hat{\gamma}_{1}$, and an estimate of the output is $\hat{y}=\varphi^{T}(k) \hat{\gamma}$. Second, form

$$
\begin{aligned}
z(k) & =\hat{y}(k)-\hat{y}_{l}(k)-\hat{\gamma}_{0}, \\
\phi^{T}(k) & =\left[\left(\varphi_{1}^{T} \gamma_{1}\right)^{2} \ldots\left(\varphi_{1}^{T} \gamma_{1}\right)^{m}\right]^{T} .
\end{aligned}
$$

The polynomial coefficients are then found as

$$
\hat{\mathbf{d}}=\arg \min _{\mathbf{d}} \sum_{k=1}^{K}\left|z(k)-\phi^{T}(k) \mathbf{d}\right|^{2} .
$$

Note that when the underlying system is not within the class of polynomial Wiener models but rather a general Wiener model parameterized in the form of a standard $\mathrm{VL}$ model as (8), then applying the procedure described above can be interpreted as projecting the nonlinearity onto a polynomial basis. This results in an additional approximation error due to the model mismatch. In [9], an iterative scheme for reducing the bias induced by said mismatch is presented.

\section{E. The Particle Filter}

The particle filter (PF), aka Sequential Monte Carlo, is a nonlinear Bayesian state estimation method [1]. Monte-Carlo simulation is used in the PF to approximate the posterior filtering distribution $p\left(x_{k} \mid y_{1: k}, u_{1: k}\right)$ of the state-space model

$$
\begin{aligned}
x(k+1) & =f(x(k), u(k))+v(k), \\
y(k) & =h(x(k), u(k))+e(k) .
\end{aligned}
$$

The approximation can be made arbitrarily accurate by increasing the number of particles. This, however, comes with an additional computational cost, limiting the number of states that can efficiently be estimated, as the number of particles needed to achieve a given resolution in the state space increases with the dimension.

One popular implementation of the PF is the Sequential Importance Resampling (SIR) algorithm. Let $x^{(i)}$ denote particle $i, w^{(i)}$ its corresponding weight, and $M$ the number of particles. Following [14], the estimation algorithm is

$$
\begin{aligned}
& \tilde{x}_{k+1}^{(i)}=f\left(x_{k}^{(i)}, u_{k}\right)+v_{k}^{(i)}, \\
& \tilde{w}_{k+1}^{(i)}=w_{k}^{(i)} p_{e}\left(y_{k}-h\left(\tilde{x}^{(i)}, u_{k}\right)\right), \\
& w_{k+1}^{(i)}=\tilde{w}_{k+1}^{(i)} / \sum_{j=1}^{M} \tilde{w}_{k+1}^{(j)},
\end{aligned}
$$

for $i=1,2, \ldots, N$. The particles are then resampled by drawing $M$ new particles $\left\{x_{k+1}^{(i)}\right\}_{i=1}^{M}$ with replacement, so that $\operatorname{Pr}\left(x_{k+1}^{(i)}=\tilde{x}_{k+1}^{(i)}\right)=w_{k+1}^{(i)}$. When the new particles have been resampled, all weights are set to $w_{k+1}^{(i)}=1 / M$.

\section{EXPERIMENTS AND DATA COLLECTION}

The experimental setup consisted of a computer screen and a video-based eye tracker from SmartEye AB, Sweden. The eye tracker records the gaze position of the test subject, i.e. the point on the screen where the subject is looking. Test subjects were placed about $50 \mathrm{~cm}$ from the computer screen, and a stimulus constituting of a colored dot moving on a black background was displayed. The stimuli, acting as input signals to the SPS, were generated using the method in [6] to provide excitation both in amplitude and frequency, and the gaze position was sampled at $60 \mathrm{~Hz}$. For the application in hand, it implies that the dot has to visit all the areas within the stimuli window and move within a suitable acceleration range, with regard to the bandwidth of the SPS.

Two groups of test subjects are considered. One control group consisting of 22 healthy individuals from 50 to 76 years old, and one group of 7 individuals diagnosed with Parkinson's disease (PD), from 61 to 80 years old. In this paper, however, data from one subject in each group are considered, with 38 measurements from the patient, and 24 measurements from the control subject. Furthermore, in the experiments with synthetic data, the same input signals (visual stimuli) are used as in the clinical trials.

Prior to the first eye-tracking test, each patient received a dose of an anti-PD drug, after an 8 hours long washout. The administered dose was set to $150 \%$ of the patient's usual morning dose of medication. This experimental protocol allows to track the patient's symptoms, as they transit from off-state to normal mobility and/or dyskinesia and back. Thus, the measurements from the patient group exhibit a range of behaviors, including lack of visible PD symptoms. Because of this, a larger domain of coefficients is expected to be needed to describe the SPS dynamics the patient group, compared to that in the control group. The distribution of model coefficients estimated for the patients is anticipated to partially overlap with those for the control group.

The tests were conducted at CTC (Clinical Trials Consultants AB) Center at the University Hospital in Uppsala, Sweden, between May and August 2015, see [17] for details.

\section{WIENER MODELING OF SMOOTH PURSUIT}

Two model structures are considered to capture the response of SPS to the visual stimuli. A VL model with $N=2$ and the kernels parametrized in the three first Laguerre functions is referred to as the full model and is written as

$$
\begin{aligned}
y(k)=y_{0} & +\sum_{j=0}^{2} \gamma_{1}(j) \psi_{j}(k)+ \\
& +\sum_{j_{1}=0}^{2} \sum_{j_{2}=j_{1}}^{2} \gamma_{2}\left(j_{1}, j_{2}\right) \psi_{j_{1}}(k) \psi_{j_{2}}(k) .
\end{aligned}
$$

A reduced model based on (20) is also considered

$$
\begin{aligned}
y(k) & =y_{0}+\gamma_{1}(0) \psi_{0}(k)+\gamma_{1}(1) \psi_{1}(k)+ \\
& +\gamma_{2}(0,0)\left(\psi_{0}^{2}(k)+\theta_{02} \psi_{0}(k) \psi_{2}(k)+\theta_{22} \psi_{2}^{2}(k)\right)
\end{aligned}
$$


Distribution of NMSE
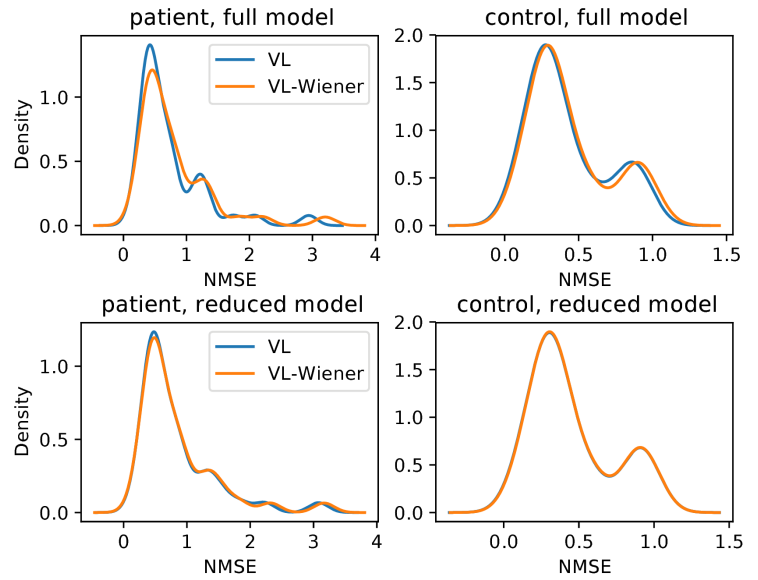

Fig. 1. Distributions of NMSE using the full and reduced VL models and WM. The error distributions are almost identical in both cases.

where $\theta_{02}=-1.81468$ and $\theta_{22}=.938454$. Model structure (21) was produced by the SPICE algorithm for sparse estimation [18] combined with principal component analysis (PCA) to extract a parsimonious model structure from (20). The data sets described in Section III were used, and sparse VL models with $N=2$ were estimated. PCA was used to identify possible functional relations between the coefficients, and a reduced model structure was selected. The VL-model reduction procedure is detailed in [2].

While (20) is parametrized by ten coefficients altogether, (21) has only four coefficients. Yet, as shown further, the modeling quality in terms of the output error is preserved with the use of the reduced model.

In view of these models, the nonlinear part is approximated by a third-order polynomial $f(x)=x+d_{2} x^{2}+d_{3} x^{3}$ to align with the structure of the Wiener model in (12).

\section{A. Identification of SPS}

For one patient diagnosed with PD and one healthy control subject, the coefficients of the Wiener model were estimated using the two-step LS approach described in Section II-D. In Fig. 1, the distributions of the normalized MSE (NMSE) for the patient and control subject are shown, in the full model case and reduced model case, respectively. Evidently, the output error does not increase due to approximating the nonlinearity by a third-order polynomial. The error distributions are almost identical in the reduced model case. As the distributions of the polynomial coefficients in Fig. 2 show, the coefficient variances for the patient is much larger than those for the control subject. The WM residuals are distributed with heavier tails than a normal one, see Fig. 3.

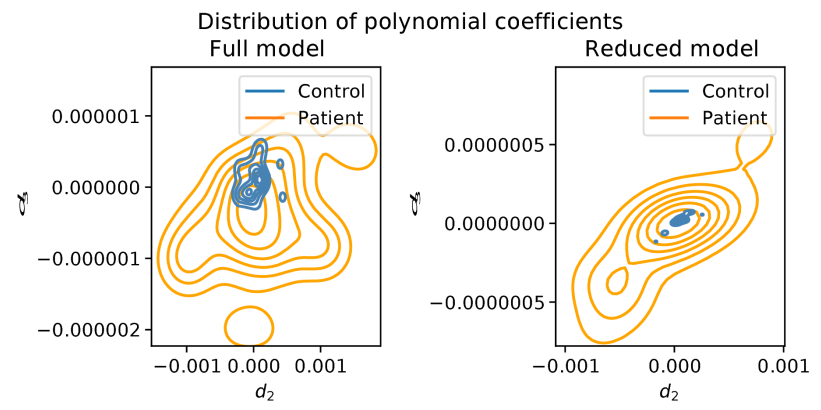

Fig. 2. Distributions of $d_{2}$ and $d_{3}$, using the full and reduced WM.
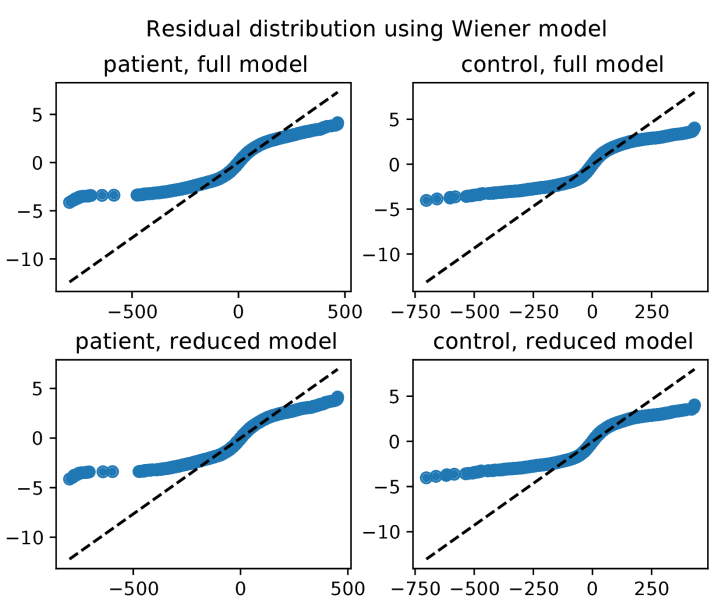

Fig. 3. Residual distributions using the full and reduced Wiener models.

\section{WIENER ESTIMATION USING THE PARTICLE FILTER}

To estimate the parameters of system (12) by a PF, an augmented state-space model is formulated as

$$
\begin{aligned}
{\left[\begin{array}{c}
\psi(k+1) \\
\mathbf{c}(k+1) \\
\mathbf{d}(k+1)
\end{array}\right] } & =\left[\begin{array}{ll}
\mathbf{F} & \mathbf{0} \\
\mathbf{0} & \mathbf{I}
\end{array}\right]\left[\begin{array}{l}
\psi(k) \\
\mathbf{c}(k) \\
\mathbf{d}(k)
\end{array}\right]+\left[\begin{array}{c}
\mathbf{G} \\
\mathbf{0}
\end{array}\right] u(k)+v(k) \\
y_{l}(k) & =\psi^{T}(k) \mathbf{c} \\
y(k) & =y_{l}(k)+d_{2} y_{l}(k)^{2}+ \\
& +d_{3} y_{l}(k)^{3}+y_{0}+\varepsilon(k)
\end{aligned}
$$

where $v_{k} \in \mathbb{R}^{7}$ and $e_{k} \in \mathbb{R}$ are white Gaussian noise sequences. Thus, the parameter states modeled as random walk are estimated simultaneously with the dynamical states, i.e. the Laguerre filter outputs.

\section{A. Synthetic data}

To test the feasibility of the concept, synthetic data were generated by simulating output of WM (12), with the parameters $\mathbf{c}=[-0.3,0.6]^{T}, \mathbf{d}=\left[d_{2}, d_{3}\right]^{T}=\left[5 \cdot 10^{-4}, 5 \cdot 10^{-7}\right]^{T}$, and $y_{0}=-9$. These parameter values are chosen to render dynamics similar to those actually observed in the SPS. Gaussian white noise was added to the simulated output resulting in a SNR of $20 \mathrm{~dB}$. The same input signals were used as in the clinical experiments. 
Parameter estimates, unscaled data
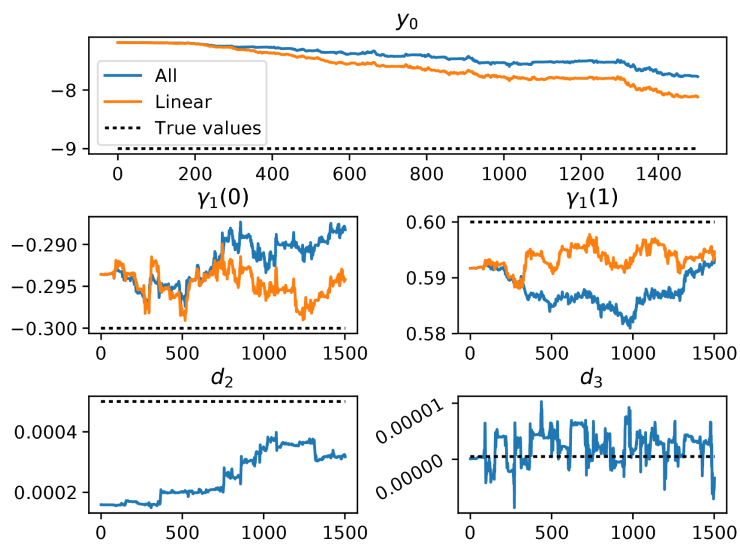

Fig. 4. Parameter estimates for unscaled data when all parameters and states are estimated (All), only the linear part and the Laguerre filter outputs are estimated (Linear), together with the true values. The estimates of the linear coefficients are close to the true values, while the polynomial coefficients are not estimated well as. There is a slight bias in the estimate of the constant.

Three scenarios were examined: estimating all states, estimating only the linear VL part, and estimating only the Laguerre filter outputs (i.e. tracking the output, given the true model). The computations were performed directly on the simulated data, as well as with the input and output signals scaled to constrain the output values to $y \in[-1,1]$. This scaling keeps the linear parameters intact but yields larger polynomial coefficients. Thus, numerical issues with estimating very small values may be avoided. Furthermore, three different input signals (visual stimuli sets) were used in order to examine whether the differences in excitation matter.

The parameter estimates at each PF iteration when using the original signals are shown in Fig. 4, while Fig. 5 depicts the parameter estimates with signal scaling. Note that the parameter estimates have been re-calculated to the original scale, to facilitate comparison. In general, the linear parameter estimates are close to the true values, both when using the scaled and unscaled data. These estimates are also very similar both in value and in the trajectory of the estimate over time, between the two cases. The true polynomial coefficients are not found, indicating that the contribution from the nonlinear part is small.

The output, however, is tracked very well, even when the parameter estimates are off. Table I shows the NMSE in the different estimation cases, where the scaled signals were scaled back to allow for comparison. There is only little difference between the three estimation cases, while the output errors differ approximately by a factor $2-5$ between the scaled and unscaled case. Note here, that the errors are small, compared to the estimates obtained from real data (see Fig. 1). Furthermore, there is no significant difference in the output error between the different input signals.

\section{B. Experiments with eye-tracking data}

A PF with $M=10^{6}$ was used to estimate the parameters of a WM for three test subjects: two controls and one patient.

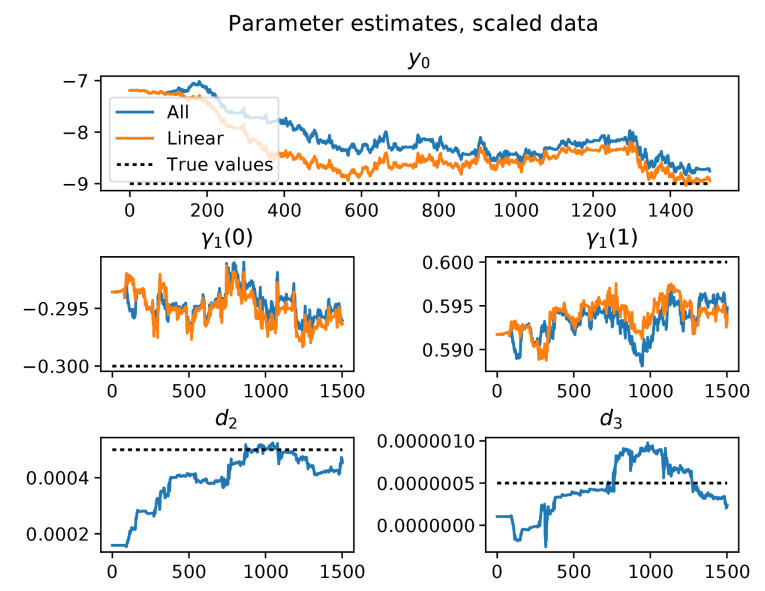

Fig. 5. Parameter estimates for scaled data when all parameters and states are estimated (All), only the linear part and the Laguerre filter outputs are estimated (Linear), together with the true values. The linear coefficients' estimates are close to the true values. The polynomial coefficients are estimated with small errors and the estimate of the constant is biased.

TABLE I

NMSE FOR PF ESTIMATION

\begin{tabular}{l|cc} 
Estimated states & Unscaled & Scaled \\
\hline \multicolumn{2}{c}{ Input signal 1 } \\
\hline All & $2.43 \cdot 10^{-3}$ & $1.03 \cdot 10^{-3}$ \\
Linear part \& output & $0.60 \cdot 10^{-3}$ & $0.34 \cdot 10^{-3}$ \\
Output only & $8.22 \cdot 10^{-8}$ & $0.96 \cdot 10^{-8}$ \\
\hline \multicolumn{3}{c}{ Input signal 2 } \\
\hline All & $1.75 \cdot 10^{-3}$ & $0.57 \cdot 10^{-3}$ \\
Linear part \& output & $0.76 \cdot 10^{-3}$ & $0.37 \cdot 10^{-3}$ \\
Output only & $1.05 \cdot 10^{-7}$ & $0.15 \cdot 10^{-7}$
\end{tabular}

Three and two data sets from the control subjects were used, respectively, and three data sets from the patient. Two of the patient data sets were recorded in an unmedicated state (after overnight wash-out, and after the medication effect had ceased, respectively), and one corresponds to the time instant when the anti-PD medication had full effect.

The estimates of the linear coefficients over time are shown in Fig. 6. There are large variations between the test subjects, while the intra-subject variations are significantly smaller. Here, no effect of disease or medication is seen in the linear part. In Fig. 7, the estimates of the Wiener polynomial coefficients are depicted. The estimates of the coefficient $d_{2}$ for the Parkinson's patient are lower than those for the controls, both in the medicated and unmedicated state. However, the estimates of $d_{3}$ differ significantly from those of the control subjects only in the unmedicated cases. The particle distributions of the PF in the last iteration are shown in Fig. 8. These distributions are instrumental in assessing the uncertainty of the parameter estimates. Evidently, the distributions overlap, yet there is a visible difference between the subjects. Also noteworthy is the fact that the uncertainty seems to be larger in the unmedicated state than under medication, as the distributions in the unmedicated state are wider and in some cases multimodal. 

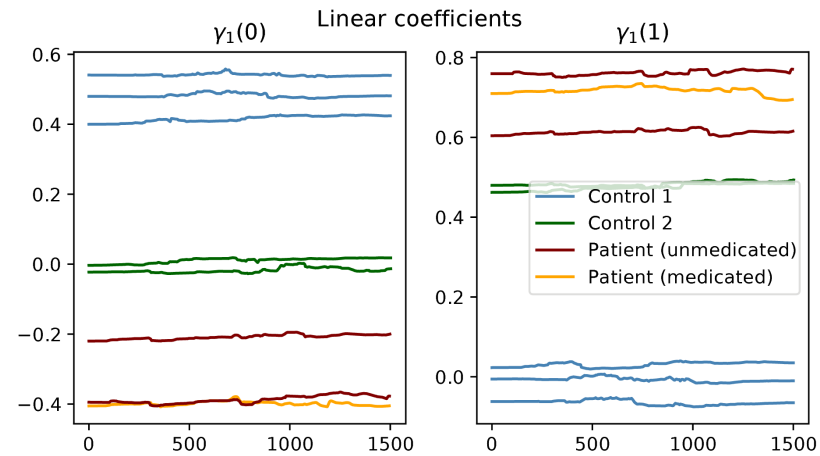

Fig. 6. Estimate trajectories of linear coefficients. Inter-patient variability is large in comparison to intra-patient variability. The linear coefficients do not seem to capture the effect of PD on SPS.

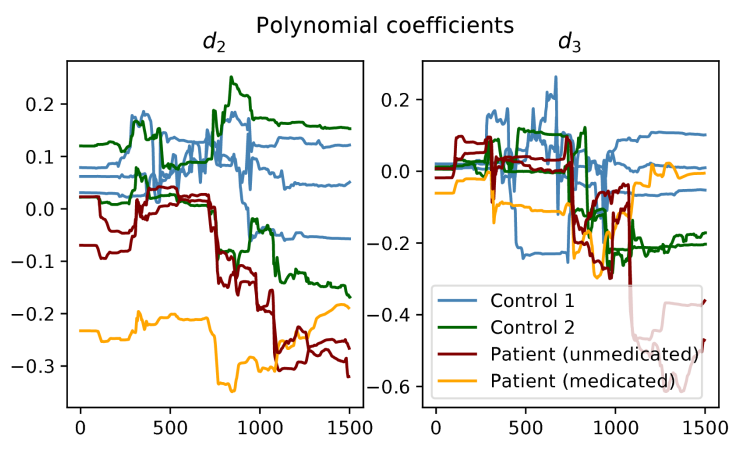

Fig. 7. Estimate trajectories of Wiener polynomial coefficients. The values for the quadratic coefficient, $d_{2}$, differ between the patient and the controls. The controls together with the medicated patient form one group with higher $d_{3}$ values, and the unmedicated patient forms another, with lower $d_{3}$ values. This indicates that the nonlinear part may capture the effects of PD on SPS.

\section{CONCLUSIONS}

The PF approach has been applied for estimation of the parameters in a polynomial Wiener model of the human SPS. Experiments on synthetic data show the potential performance of the approach. Experiments on eye-tracking data suggest that the uncertainty of the parameter estimates increase with symptoms of Parkinson's disease. There is also some indication that symptoms of Parkinson's disease can be captured by the nonlinear part of the Wiener model.

The results presented should be viewed as indications, and future work needs to be done on two fronts. First, larger datasets must be considered, to be able to generalize the results further. Second, the variance of the driving noise in the parameter proposals may be tuned further, and better parameter estimates could possibly be found.

\section{REFERENCES}

[1] M. S. Arulampalam et al., "A tutorial on particle filters for online nonlinear/non-Gaussian Bayesian tracking," IEEE Transactions on Signal Processing, vol. 50, (2), pp. 174-188, 2002.

[2] V. Bro and A. Medvedev. "Constrained SPICE in Volterra-Laguerre Modeling of Human Smooth Pursuit." IEEE Conference on Control Technology and Applications, Hawaii, 2017.

[3] J. M. Gibson, R. Pimlott and C. Kennard, "Ocular motor and manual tracking in Parkinson's disease and the effect of treatment," Journal of Neurology Neurosurgery and Psychiatry, vol. 50, (7), pp. 853-860, 1987.

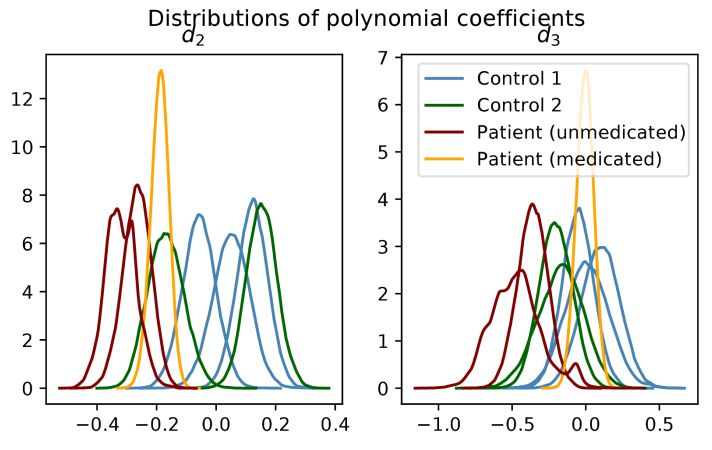

Fig. 8. Distributions of particles for the Wiener polynomial coefficients in the last iteration of PF. While there is overlap between the distributions, the two groups can be seen, with the exception of one data set from a control subject. Note that the width of the distribution is considerably reduced in the patient's medicated state, compared to the unmedicated state.

[4] D. Jansson et al., "Mathematical modeling and grey-box identification of the human smooth pursuit mechanism," IEEE International Conference on Control Applications (CCA), Yokohama, Japan, 2010.

[5] D. Jansson and A. Medvedev, "Dynamic smooth pursuit gain estimation from eye tracking data," 50th IEEE Conference on Decision and Control and European Control Conference (CDC-ECC), Orlando, FL, 2011.

[6] D. Jansson and A. Medvedev, "Visual stimulus design in parameter estimation of the human smooth pursuit system from eye-tracking data," in American Control Conference (ACC), Washington, DC, 2013.

[7] D. Jansson, O. Rosen and A. Medvedev, "Non-parametric analysis of eye-tracking data by anomaly detection," Control Conference (ECC), 2013 European, 17-19 July 2013.

[8] D. Jansson et al., "Smooth pursuit in Parkinson's disease is nonlinear but remains dominantly linear in healthy aging,", MDS 18th International Congress of Parkinson's Disease and Movement Disorders, Volume 29, June 2014, Abstract Supplement.

[9] D. Jansson and A. Medvedev, "Identification of polynomial Wiener systems via Volterra-Laguerre series with model mismatch," IFACPapers on line, vol. 28, (11), pp. 831-836, 2015.

[10] A. H. Jazwinski, Stochastic Processes and Filtering Theory. New York: Academic Pr, 1970.

[11] S. Marino et al., "Quantitative Analysis of Pursuit Ocular Movements in Parkinson's Disease by Using a Video-Based Eye Tracking System," European Neurology, vol. 58, (4), pp. 193-197, 2007.

[12] A. Medvedev et al., "Oscillations-free PID control of anesthetic drug delivery in neuromuscular blockade," Computer Methods and Programs in Biomedicine, 2016.

[13] G. A. O'Driscoll and B. L. Callahan, "Smooth pursuit in schizophrenia: A meta-analytic review of research since 1993," Brain and Cognition, vol. 68, (3), pp. 359-370, 2008.

[14] B. Ristic, S. Arulampalam and N. Gordon, Beyond the Kalman Filter: Particle Filters for Tracking Applications. Boston, MA, London; Artech House, 2004.

[15] O. Rosen et al., "Efficient Parallel Implementation of State Estimation Algorithms on Multicore Platforms," IEEE Transactions on Control Systems Technology, vol. 21, (1), pp. 107-120, 2013.

[16] Schön, Thomas B. "Nonlinear system identification using particle filters." Encyclopedia of Systems and Control, pp. 882-890, 2015.

[17] M. Senek et al., "Levodopa/carbidopa microtablets in Parkinson's disease: a study of pharmacokinetics and blinded motor assessment," European Journal of Clinical Pharmacology, vol. 73, (5), pp. 563-571, 2017.

[18] P. Stoica et al., "SPICE and LIKES: Two hyperparameter-free methods for sparse-parameter estimation," Signal Processing, vol. 92, (7), pp. $1580-1590,2012$.

[19] Thier, Peter, and Uwe J. Ilg. "The neural basis of smooth-pursuit eye movements." Current opinion in neurobiology, 15, (6), 645-652, 2005.

[20] I. M. S. Wilkinson, "The influence of drugs and alcohol upon human eye movement," Proceedings of the Royal Society of Medicine, vol. 69, (7), pp. 479-480, 1976. 